\title{
Revisión
}

\section{Hermafroditismo en peces teleósteos y sus implicaciones en la acuacultura comercial}

Hermaphroditism in teleost fishes and their implications in commercial aquaculture

\author{
Jaime Navarro-Flores ${ }^{1}$, Leonardo Ibarra-Castro ${ }^{2 *}$, Juan M. Martinez-Brown ${ }^{2,3}$, Oscar \\ Iram Zavala-Leal ${ }^{1,4}$
}

\author{
'Posgrado en Ciencias Biológico Agropecuarias, Universidad Autónoma de Nayarit, Ciudad de la Cultura "Amado Nervo" Tepic, \\ Nayarit, México, C.P. 63155 \\ ${ }^{2}$ Laboratorio de Reproducción y Cultivo Larvario de Peces Marinos, Centro de Investigación en Alimentación y Desarrollo A.C., Unidad \\ Mazatlán, Ave. Cerritos S/N, C.P. 82010, A.P. 711, Mazatlán, Sinaloa, México \\ ${ }^{3}$ Dirección de Cátedras-CONACYT, Consejo Nacional de Ciencia y Tecnología (CONACYT), Ciudad de México, México \\ ${ }^{4}$ Escuela Nacional de Ingeniería Pesquera, Universidad Autónoma de Nayarit, Bahía de Matanchén Km. 12, Carretera a Los Cocos, \\ San Blas, Nayarit, México, C.P. 63740 \\ *Autor de correspondencia: leonardo.ibarra@ciad.mx; leobeis@hotmail.com
}

\begin{abstract}
The teleost fishes are the most diverse group of vertebrates and present a great variety in their reproduction form. Gonocorism is the most common form of reproduction among fish. However, a significant number of fish present hermaphroditism, that is, the same individual presents both sexes, simultaneously or sequentially. The point in time at which the sex change occurs is not necessarily instantaneous and can be interpreted at many different levels (by social, morphological and age behavior). The mechanisms of sexual determination and sexual differentiation are involved in this sex change and may be affected by the genetic inheritance or by the environmental changes in which an individual finds itself. The activity aromatase, estradiol, 11-ketotestosterone and testosterone are involved in the regulation of the reproductive process of hermaphroditic females and males. The pre-optic area (POA) and the hypothalamus are the two regions of the brain responsible for the control of the reproductive physiology and reproductive behavior of fish. Within the endocrine status of sequential hermaphroditic fish, great similarities with the endocrinology of gonochoric species are observed. Taming hermaphroditic species for reproductive purposes can bring great complications; the most common is to keep the desired sex ratio constant, therefore, the control of sex is a priority. To achieve this, it is necessary to know the reproductive physiology of the selected species and implement some strategy to manipulate this sex change, either through social factors or hormonal therapies.
\end{abstract}

Key words: Reproduction, sex change, endocrinology, hermaphroditism

\begin{abstract}
Resumen.- Los peces teleósteos son el grupo más diverso de vertebrados y presentan gran variedad de formas de reproducción. El gonocorismo es la forma más común de reproducción entre los peces. Sin embargo, un número significativo de peces presenta hermafroditismo, es decir, en el mismo individuo se encuentran ambos sexos de manera simultánea o secuencial. El punto en el tiempo en el cual ocurre el cambio de sexo no es necesariamente instantáneo y puede ser interpretado en muchos niveles diferentes (por comportamiento social, morfológico y por edad). Los mecanismos de determinación y diferenciación sexual están involucrados en el cambio de sexo y pueden verse afectados por la herencia genética o por cambios ambientales en los que se encuentre el individuo. La actividad aromatasa, estradiol, 11-ketotestosterona y testosterona están involucrados en la regulación del proceso reproductivo de hembras y machos hermafroditas. El área pre-óptica (POA) y el hipotálamo son las dos regiones del cerebro encargadas del control de la fisiología reproductiva y comportamiento reproductivo de los peces. Dentro del estatus endocrino de peces hermafroditas secuenciales, se observan grandes similitudes con la endocrinología de especies gonocóricas. El domesticar especies hermafroditas con fines reproductivos, puede traer consigo grandes complicaciones, la más común es mantener constante la proporción de sexos, por lo tanto, el control del sexo es una prioridad. Para lograrlo es necesario conocer la fisiología reproductiva de la especie seleccionada e implementar alguna estrategia para manipular ese cambio de sexo, ya sea mediante factores sociales o terapias hormonales.
\end{abstract}

Palabras clave: Reproducción, cambio de sexo, endocrinología, hermafroditismo

\section{INTRODUCCIÓN}

Los peces hermafroditas son organismos que presentan ambos sexos en un mismo individuo. De las 33000 diferentes especies de peces (Avise 2011), aproximadamente el $2 \%$ presenta un tipo de hermafroditismo (Sadovy de Mitchenson \& Liu 2008), simultaneo o secuencial. Este último es el tipo más común entre los peces que habitan en zonas de arrecifes como el barramundi Lates calcarifer (Guiguen et al. 1993), la dorada Sparus aurata (Zohar et al. 1978), el róbalo blanco Centropomus undecimalis (Taylor et al. 2000), meros Epinephelus tauvina (Tan \& Tan 1974) y E. morio (Brulé et al. 1999), entre otros.
Respecto a los factores fisiológicos, endocrinos y ambientales que influyen en los procesos de determinación y diferenciación sexual que presentan los peces teleósteos, estos han sido revisados y discutidos ampliamente por Nakamura et al. (1998), Baroiller et al. (1999), Devlin \& Nagahama (2002), y Pandian (2011) de forma independiente. Mientras que las etapas del desarrollo gonadal en peces teleósteos han sido descritas por Wallace \& Selman (1990), Bromage \& Cumaranatunga (1988) y Taylor et al. (1998). Conociendo la estrategia y biología reproductiva de una especie con potencial para acuacultura, se pueden diseñar planes de trabajo para la formación y renovación de sus 
lotes de reproductores en condiciones controladas, como es el caso de los espáridos Acanthopagrus schlegelii y la dorada Sparus aurata, especies que son cultivadas a nivel comercial (Pavlidis \& Mylonas 2011). Desde el año 2000, en América Latina, se ha aumentado el interés en cultivar diversas especies marinas, entre ellas especies hermafroditas pertenecientes a la familia Centropomidae, particularmente conocidos como róbalos (Centropomus sp.) (AlvarezLajonchére \& Tsuzuki 2008) y los meros pertenecientes a la familia Serranidae y género Epinephelus (Bell \& Gervis 1999). Sin embargo, la información disponible para alcanzar este objetivo es escasa o completamente nula. Por lo tanto, el objetivo de la presente revisión se enfocó en la detección de los problemas que representa el control de la reproducción de especies hermafroditas en condiciones de cautiverio para fines de cultivo. El análisis de esta información facilitará el entendimiento de aquellos interesados en el cultivo de especies hermafroditas con importancia comercial y de esparcimiento recreativo.

\section{PATRONES de REPROdUCCIÓN EN PECES TELEÓSTEOS MARINOS}

Dentro del grupo de vertebrados, los peces teleósteos presentan una gran diversidad en sus formas de reproducción. La mayoría de los peces son iteróparos (que se reproducen más de una vez durante su ciclo de vida). Sin embargo, otros peces como el salmón coho (Oncorhynchus kisutch), son semélparos (que solo presentan un evento reproductivo y mueren). El gonocorismo (sexos separados) es el patrón sexual más común en los peces teleósteos, donde todos los individuos maduran sexualmente y se reproducen exclusivamente como machos o como hembras durante toda su vida. Sin embargo, a diferencia de la mayoría de los vertebrados, un número significativo de peces funciona como hembra o macho durante una parte de su ciclo de vida (hermafroditas). El hermafroditismo funcional se presenta en 27 familias, con dos patrones principalmente: simultáneo y secuencial. El hermafroditismo simultáneo, implica que los peces funcionan al mismo tiempo como hembras y machos en un mismo periodo de desove, es decir, sus gónadas contienen simultáneamente tejido testicular y tejido ovárico maduro. Pandian (2010) clasificó el hermafroditismo simultaneo en tres tipos: 1) Hermafroditas con auto-fertilización, y es representada por la especie Rivulus marmoratus y algunas especies del género Serranus; 2) hermafroditas que intercambian sus gametos, por ejemplo: Serranus tortugarum y Hypoplectrus nigricans, estas especies tienen la particularidad de desovar solo en parejas, uno es el iniciador (libera los huevos) y el otro el seguidor (fertilizador); y 3 ) hermafroditas con potencial de autofertilización, a este lo compone la familia Chlorophthalmidae (género Chlorophthalmus), Scopelarchidae (Bathypterois), Bathysauridae (Bathysaurus) (Sadovy de Mitchenson \& Liu 2008, Pandian 2010). El hermafroditismo secuencial ha sido reportado en 350 especies, pertenecientes a 34 familias (Sadovy de Mitchenson \& Liu 2008). Se clasifica en hermafroditismo: protándrico, protogínico y serial o bidireccional. El hermafroditismo secuencial protándrico, primero maduran sexualmente como machos por espacio de 2 a 3 temporadas reproductivas, según la especie y después cambian a hembras manteniéndose en este sexo el resto de su vida reproductiva. Esta forma de hermafroditismo es menos común, ya que solo ocurre en 21 géneros repartidos en 6 familias [C. undecimalis (Taylor et al. 2000), L. calcarifer (Guiguen et al. 1994), A. schlegelii (Chang et al. 1994)]. Los hermafroditas protogínicos, primero maduran sexualmente como hembras y después cambian a machos (Avise \& Mank 2009). La familia Serranidae, a la que pertenece la gran mayoría de meros presentan este tipo de hermafroditismo al igual que algunas especies de la familia Sparidae, como Pagellus erythrinus (Basurco et al. 2011). Finalmente, el hermafroditismo serial o bidireccional, se caracteriza por cambiar de sexo y/o alternar entre los sexos más de una vez, asegurando así un mayor éxito reproductivo, como Paragobiodon echinocephalus (Munday et al. 1998).

El hermafroditismo se presenta con mayor frecuencia en los peces que habitan las profundidades de los océanos, en donde las densidades de la población son relativamente bajas y encontrar una pareja para reproducirse se vuelve un problema común. Sin embargo, la mayoría de los peces hermafroditas simultáneos y secuenciales se les asocia con los arrecifes de coral (Pandian 2010, Avise 2011). En muchos de los casos, el cambio del sexo se debe a factores sociales, como la desaparición de un macho o una hembra del mismo harem o cardumen. Lo anterior revela, que estos fenómenos del cambio de sexo y que los mecanismos de determinación sexual en peces son más diversos y plásticos, a diferencia de los vertebrados superiores.

\section{DETERMINACIÓN SEXUAL}

La determinación sexual es el mecanismo genético o ambiental por el cual se define el sexo de un individuo, para que este sea macho o hembra (Wootton \& Smith 2015). Por lo tanto, los procesos de determinación sexual pueden ser controlados por la información genética que hereda un individuo o puede darse en respuesta a los cambios de las condiciones ambientales en las que se encuentre un individuo. En la mayoría de los vertebrados como los mamíferos y aves, el sexo se determina por los cromosomas sexuales tan pronto se forma el cigoto resultante de la concepción (determinación sexual genética, GSD) (Lee et al. 2001). Mientras que en los peces hay muchas excepciones a esta regla, donde el desarrollo final de la gónada puede estar influido por fluctuaciones en los factores intrínsecos, como el crecimiento, o por factores ambientales extrínsecos como la temperatura, $\mathrm{pH}$, salinidad, hormonas endocrinas o contaminación en el medio acuático (Lee et al. 2001, Devlin \& Nagahama 2002). 
A diferencia de otros vertebrados, en peces el mecanismo de determinación sexual es considerado diverso y más plástico (Pandian 2011). Esto origina la flexibilidad necesaria para la reorganización gonadal y puede proceder en conjunto con los factores intrínsecos y ambientales. Entonces, la determinación del sexo en un pez puede ser influida por un proceso genético o por cualquier efecto de variación ambiental o fisicoquímico.

Normalmente, la determinación del sexo en vertebrados se divide en dos sistemas, los cuales pueden ser aplicados en peces: la GSD y determinación sexual ambiental (ESD). La ESD, se presenta principalmente en reptiles, anfibios, y en algunos peces (70 especies de teleósteos), donde el sexo es fisiológicamente determinado por los factores ambientales (temperatura, $\mathrm{pH}$, salinidad), después de días o semanas de la fertilización y cualquiera de las variables anteriores puede ser la causante de la determinación sexual (Baroiller et al. 1999). En el caso de los peces diferenciados, el sexo se determina después de la eclosión y durante el desarrollo larvario y post-larvario en los peces que son sexualmente indiferenciados (Piferrer 2009).

Particularmente, la temperatura del agua ha demostrado ser uno de los factores ambientales clave que influye en el sexo fenotípico de muchas especies de teleósteos comercialmente importantes como la trucha arcoíris (Oncorhynchus mykiss) (Valdivia et al. 2014), medaka (Oryzias latipes) (Hayashi et al. 2010), pez cebra (Danio rerio) (Abozaid et al. 2012), pejerrey (Odontesthes bonariensis) (Struussmann et al. 1996) y pargo flamenco (Lutjanus guttatus) (datos no publicados) en donde la alta temperatura del agua de cultivo puede aumentar significativamente la tasa de masculinización. Esto podría tener un efecto en la proporción de sexos a obtener en una producción masiva de juveniles, lo cual puede influir en la proporción de tallas durante las primeras etapas del cultivo y por ende en la dinámica de crecimiento durante el proceso de engorda. Lo deseable es cultivar aquel sexo que tenga mayor tasa de crecimiento y que retarde su madurez sexual, para evitar pérdidas de energía que no sean dirigidas al crecimiento. Por lo tanto, las especies con potencial acuícola deben de ser estudiadas en varios aspectos de su biología, para conocer cuál de los dos sexos puede tener mejor desempeño en el cultivo y determinar cuál de las técnicas genéticas, ambientales u hormonales, son mejores para producir el sexo deseado (Piferrer 2009).

\section{DIFERENCIACIÓN SEXUAL}

La diferenciación sexual se define como el proceso ontogenético por el cual una gónada indiferenciada se define o madura sexualmente como ovario o testículo y son generados por una cascada de regulaciones genéticas o ambientales (Piferrer \& Guiguen 2008). El proceso reúne todos los acontecimientos que tienen lugar en el primordio gonadal, incluyendo la migración de células germinales primordiales (PGC) y el establecimiento de las crestas gonadales (Piferrer 2001). En las gónadas es donde se lleva a cabo el proceso de gametogénesis generando gametos haploides como los óvulos en la gónada femenina y espermatozoides en la gónada masculina. En los peces la diferenciación sexual es un proceso lábil y flexible sometido a la inducción natural y artificial del cambio de sexo/ inversión mediante genética, endocrino y/o manipulaciones ambientales (Pandian 2013). El control endocrino de la diferenciación sexual implica una compleja interacción entre el cerebro y gónada a través de la producción de gonadotropinas hipofisarias y derivados de esteroides que se producen en la gónada y el cerebro (Devlin \& Nagahama 2002). Los esteroides sexuales particularmente andrógenos y estrógenos juegan un papel importante en la regulación del proceso de la diferenciación sexual, ya que actúan como hormonas endocrinas para influir en otros tipos de células y órganos que intervienen en la determinación del sexo (Devlin \& Nagahama 2002, Piferrer \& Guiguen 2008). Esto se ha corroborado mediante el uso de estrógenos exógenos, que pueden afectar directamente la diferenciación gonadal obteniendo un ovario funcional, mientras que los tratamientos con andrógenos pueden llegar a masculinizar hembras genéticas (Piferrer 2001, Yaron \& Sivan 2005).

\section{ENDOCRINOLOGÍA GENERAL Y EL CAMBIO DE SEXO EN PECES MARINOS}

\section{Endocrinología durante el proceso de REPRODUCCIÓN EN HEMBRAS}

Durante la vitelogénesis la hormona folículo estimulante (FSH) o hormona luteinizante ( $\mathrm{LH}$ ) estimulan la producción de testosterona $(\mathrm{T})$ por las células de la teca y posteriormente, es aromatizada a $17-\beta$ estradiol $\left(E_{2}\right)$ en la granulosa. En respuesta a esta estimulación por $\mathrm{E}_{2}$, el hígado produce vitelogenina (VTG), la cual es secuestrada por el ovocito por medio de un receptor estimulado por la FSH. Cuando la vitelogénesis finaliza, en el plasma sanguíneo aparece la LH y estimula una caída de $\mathrm{E}_{2}$ en el plasma. Entonces, se presenta un incremento transitorio de $\mathrm{T}$ en el plasma durante el proceso de la migración de la vesícula germinal y un marcado incremento en el plasma de los niveles de esteroides inductores de la maduración (MIS), los cuales actúan a nivel de las capas foliculares para inducir a la maduración final del ovocito (FOM) (Mylonas \& Zohar 2001). Los esteroides inductores a la maduración más

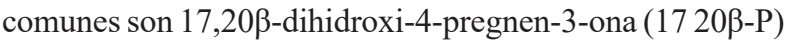

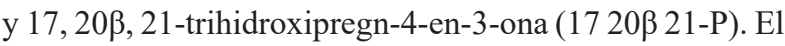
remanente de $\mathrm{E}_{2}$ induce incremento de la $\mathrm{LH}$ para que se dé la FOM, pero también la LH induce a la competencia por madurar del ovocito, un proceso por el cual, los MIS permiten al ovocito responder y experimentar la maduración final (Patiño et al. 2001). 


\section{Endocrinología durante El PROCESo de REPRODUCCIÓN EN MACHOS}

Las gonadotropinas regulan la espermatogénesis produciendo andrógenos en los testículos, principalmente $\mathrm{T}$ y 11-ketotesterona (11-KT). Como la $\mathrm{T}$ es el precursor de 11-KT, los niveles de los dos andrógenos varían durante la mayor parte de la temporada reproductiva. Las más altas concentraciones de 11-KT se alcanzan durante la espermatogénesis y empiezan a declinar durante el periodo de espermiación. En los testículos inmaduros se ha demostrado que la hormonas gonadotrópicas ( $\mathrm{GtH})$ inducen a la producción de 11-KT por las células de Leydig estimulando la producción de activina B producida por las células de Sertoli, lo cual induce a la espermatogénesis. Lo mismo que para las hembras, un incremento de los niveles de LH en el plasma sanguíneo al inicio de la temporada de desove causa el cambio en la producción de esteroides. Los andrógenos producidos por los testículos producen los MIS. La LH y los MIS estimulan la producción de plasma seminal provocando un incremento en el volumen espermático y los MIS tienen la capacidad de estimular la motilidad de los espermatozoides almacenados, por el incremento del $\mathrm{pH}$ del líquido seminal (Schulz et al. 2010). Es posible concluir que el $\mathrm{E}_{2}, 11-\mathrm{KT}$ y la T están involucrados en la regulación del proceso reproductivo de hembras y machos, por lo que estos esteroides son ampliamente ligados durante el cambio de sexo en especies hermafroditas (Frisch 2004). Patrón que se presenta en el pez payaso (Amphiprion melanopus) un hermafrodita protándrico, durante la transición de cambio de sexo (macho a hembra), el principal esteroide gonadal predominante asociado con el cambio de sexo es el $\mathrm{E}_{2}$, seguido del incremento de $\mathrm{T}$ y androstenediona (AD). En cambio en los machos maduros la 11-KT se encuentra en concentraciones más altas con respecto al $\mathrm{E}_{2}$ y $\mathrm{AD}$ (Godwin \& Thomas 1993).

\section{EL CEREBRO Y SU RELACIÓN CON LA ACTIVIDAD AROMATASA GONADAL EN EL CAMBIO DE SEXO EN ESPECIES HERMAFRODITAS}

Durante el cambio de sexo, en el cerebro se observa un incremento en el tamaño y número de células secretoras de la hormona liberadora de gonadotropinas $(\mathrm{GnRH})$. El área pre-óptica (POA) y el hipotálamo, son las dos regiones del cerebro encargadas del control de la fisiología reproductiva y del comportamiento reproductivo de los peces (Frisch 2004). Además, en el cerebro de los peces también se expresa la actividad aromatasa, que es un miembro de la superfamilia del citocromo P450 de las enzimas que cataliza la formación de estrógenos (principalmente $\mathrm{E}_{2}$ ) a partir de andrógenos (Piferrer \& Blázquez 2005).

La actividad aromatasa de diferentes especies de peces teleósteos se expresa principalmente en las gónadas como P450aromA y en el cerebro como P450aromB. También se ha detectado su expresión en otros órganos o tejidos, como la pituitaria, retina, riñón, bazo, grasa intestinal y el hígado, pero en concentraciones mucho más bajas que en las gónadas y el cerebro (Piferrer \& Blázquez 2005). La aromatasa es la responsable del paso final en la biosíntesis de estrógenos, catalizando la aromatización de $\mathrm{AD}$ y $\mathrm{T}$ en estrona y $\mathrm{E}_{2}$, respectivamente y su actividad determina la proporción de andrógeno-a-estrógeno en el desarrollo de gónadas (Shen et al. 2013).

Se ha considerado que en especies de peces gonocóricas los estrógenos son esteroides que promueven a la feminización o masculinización según sea el caso. Desde el trabajo pionero de Yamamoto (1969), quien obtuvo la feminización de organismos mediante la administración exógena de estrógenos; durante el inicio del desarrollo en peces, se han realizado un gran número de experimentos con lo cual han demostrado que los tratamientos con estrógenos son altamente efectivos en la feminización o masculinización de diferentes especies de peces (Pandian \& Sheela 1995, Piferrer 2001). Estos resultados permitieron concluir que los tratamientos con inhibidores de aromatasa (AI) (i.e., letrozol y fadrozol), bloquean la síntesis de los estrógenos, induciendo a la masculinización cuando se aplicaba durante el periodo de diferenciación sexual (periodo lábil), en especies como el salmón chinook O. tshawytscha (Piferrer et al. 1994), tilapia del nilo Oreochromis niloticus (Guiguen et al. 1999, Afonso et al. 2001), carpa común Cyprinus carpio (Tzchori et al. 2004) y el fletan del Atlántico Hippoglossus hippoglossus (Babiak et al. 2012). Los tratamientos de inversión sexual en peces se aplicaron durante el periodo de diferenciación sexual, sin embargo, respecto a la sensibilidad de los tratamientos de inversión sexual en adultos, la información es escasa, pero dichos tratamientos pueden emplearse en peces diferenciados sexualmente (Guiguen et al. 2010).

La actividad aromatasa puede ser afectada principalmente por factores ambientales como la temperatura. Lo anterior ha sido demostrado en muchas especies de peces, así como en muchos reptiles que presentan determinación sexual dependiente-temperatura (TSD) (Lance 2009). La expresión de los niveles del gen Cyp19ala durante la diferenciación sexual relaciona los efectos de la temperatura con la proporción de sexos con la alteración. Las hembras genéticas del lenguado japonés Paralichthys olivaceus cuando se exponen a temperaturas bajas o a altas temperaturas, obtienen el 100\% hembras o 100\% de machos, respectivamente (Guiguen et al. 2010). Dichos resultados indican que la exposición a altas temperaturas durante el periodo termosensible suprime la expresión del gen Cyp19ala en esta especie de pez, que probablemente da como resultado una baja actividad de la aromatasa y a su vez, bajas concentraciones de $\mathrm{E}_{2}$. Debido a esto, la falta del estrógeno provoca la supresión de la expresión del gen Cyp19al y la actividad aromatasa es la responsable de la masculinización de las hembras genéticas que fueron expuestas a altas temperaturas (Blázquez et al. 2008). 
Sin embargo, en las especies hermafroditas, las diferentes señales (ambiente social como la dominancia, proporción de sexos, señales ambientales) varían de una especie a otra y cada situación específica puede inducir cambios en el sexo. La fisiología que dirige el cambio de sexo ha recibido poca atención y se ha enfocado principalmente en la producción de esteroides, los cuales se han examinado minuciosamente durante el cambio de sexo gonadal en diversas especies y han demostrado mediar el cambio de sexo de forma natural o por inducción (Frisch 2004). La relación de los estrógenos con el papel de la aromatasa, se han analizado en diversas especies protándricas. En el barramundi L. calcarifer el incremento del $\mathrm{E}_{2}$ en el suero sanguíneo, se le asocia con el cambio de sexo de macho a hembra (Guiguen et al. 1993), al igual que en la dorada, S. aurata (Wong et al. 2006) y en A. schleglii (Chang et al. 1994). En contraste, en los hermafroditas protóginos como el mero de manchas rojas, E. akaara, las concentraciones de $\mathrm{E}_{2}$ en el plasma sanguíneo disminuyen durante la transición del cambio de sexo de hembra a macho (Li et al. 2007). En conclusión, la actividad aromatasa se detecta durante la transición del cambio de sexo en los hermafroditas protándricos y esta disminuye en los hermafroditas protóginos (Li et al. 2007). Estos procesos de reproducción y cambios de sexo pudieran ser controlados mediante el eje reproductivo de manera natural controlando el ambiente social, establecimiento de jerarquías o dominancia, proporción de sexos y señales medioambientales.

\section{INTERACCIÓN DEL EJE HIPOTÁLAMO-HIPÓFISIS EN EL CAMBIO DE SEXO DE MACHO A HEMBRA Y DE HEMBRA A MACHO}

Como ya se ha mencionado, el eje hipotálamo-hipófisisgónada (HPG) controla el comportamiento y la reproducción en los peces teleósteos, síntesis de las hormonas esteroideas y por su propia naturaleza está involucrado en el mantenimiento y/o inicio del cambio de sexo en peces hermafroditas. Sin embargo, el sistema endocrino normal también puede ser interrumpido por sustancias libres que se encuentran en el medio ambiente, a través de vías y mecanismos distintos a los mediados por los receptores esteroideos clásicos (Hinfray et al. 2006). Por lo tanto, la función del eje HPG puede ser afacetado por contaminantes, alterando la función endocrina (químicos con disrupción endocrina EDC) natural del eje reproductivo y la esteroidogénesis en general (Hamlin 2014). Kavlock et al. (1996) definieron a EDC como "un agente exógeno que interfiere con la producción, liberación, transporte, metabolismo, unión, acción o eliminación de las hormonas naturales en el organismo alterando su homeostasis y la regulación de los procesos de desarrollo".

Los peces son particularmente susceptibles a contaminantes porque están expuestos continuamente a químicos disueltos en su medio acuático, los cuales pueden permear a través de la piel, por absorción o por ingestión. Muchos de estos contaminantes se acumulan en el organismo sin poder ser degradados ni liberados, y por consecuencia ejercen sus efectos nocivos en el pez portador alterando la fecundidad y fertilidad (Colborn et al. 1993, Sun et al. 2014). Algunos de los contaminantes pueden comportarse como estrógenos, por lo cual interactúan con los receptores de estrógenos del pez causando el efecto de feminización de un pez genéticamente masculino (Hamlin 2014).

\section{COMPUESTOS CON EFECTO HORMONAL QUE ESTIMULAN AL CAMBIO DE SEXo}

Mediante diversas investigaciones realizadas en condiciones de laboratorio, utilizando diferentes especies de peces, se ha examinado el efecto de diferentes sustancias químicas como los xenobióticos antiestrogénicos, los cuales alteran la actividad de aromatasa. Esto es importante porque estos contaminantes pueden dañar el sistema reproductivo natural de especies gonocóricas y hermafroditas del medio silvestre (Mills \& Chichester 2005). Los problemas más comunes son un cambio de sexo constante en especies que no son hermafroditas y bioacumulación en el músculo, lo que puede presentar un riesgo para las poblaciones naturales de peces y de salud si los peces son para consumo humano. Un EDC puede afectar al sistema endocrino por diversas vías; pueden actuar sobre la síntesis, secreción, transporte, acción y eliminación de las hormonas endógenas (Segner et al. 2003). Una forma es la unión y activación de los receptores estrogénicos (los contaminantes actúan como estrógenos, e.g., xenoestrógenos, ecoestrógenos o estrógenos ambientales) o por la imitación del $\mathrm{E}_{2}$. Otro efecto de los compuestos xenobióticos antiestrogénicos se da por la unión, pero no activación del receptor estrogénico, es decir actúan como un anti estrógeno (Chichizola 2004). Entre los posibles biomarcadores usados para probar el efecto de estos compuestos, particularmente de los xenoestrógenos, es la producción de VTG y la expresión del gen $C Y P 1 A$, involucrado en la actividad aromatasa (Piferrer \& Blázquez 2005, Hinfray et al. 2006). Entre los efectos de los compuestos xenoestrogénicos están: inducción a la VTG y muerte celular en hepatocitos de Ameiurus nebulosus (Holland et al. 1999), inducción VTG y reducción de los niveles de esteroides en $C$. carpio y Stizostedion vitreum capturados en las cercanías de una planta de tratamiento de aguas negras (Folmar et al. 1996, 2001). Al igual que un incremento de VTG en plasma de los ejemplares machos de Platichthys flesus colectados en estuario contaminado por fábricas de gas y aceite (Allen et al. 1999).

Existe una gran cantidad de compuestos que pueden actuar como xenoestrógenos: fitoestrógenos (genisteína, cumestrol), herbicidas (alacloro, atrazina, nitrofen), insecticidas (DDT, lindano, endosulfan, dieldrina, toxafeno), fungicidas (benomilo, mancozeb, tributilo), compuestos policlorobifenilos ( $\left.\mathrm{PCB}^{\prime} \mathrm{S}\right)$, dioxinas, plásticos (ftalatos), metales pesados (plomo, mercurio, cadmio), productos domésticos (detergentes y surfactantes), productos 
farmacéuticos (dietilbestrol, etinilestradiol), entre otros (DeRosa et al. 1998, Jones et al. 2000). Para más información sobre este tema puede consultarse la revisión realizada por Mills \& Chichester (2005) y Cheshenko et al. (2008).

\section{IMPLICACIONES AL DOMESTICAR ESPECIES HERMAFRODITAS DE IMPORTANCIA COMERCIAL CON FINES DE REPRODUCCIÓN}

Normalmente, el primer lote de reproductores para cautiverio, es conformado por organismos silvestres y estos se estabulan al cautiverio por cierto tiempo antes de alcanzar la reproducción. Pueden estar en constante reproducción por 1 o 2 años dependiendo de la edad de los reproductores y tipo de hermafroditismo que presente la especie. Por ejemplo: S. aurata, A. schlegelii, P. bogaraveo, L. calcarifer y $C$. viridis son hermafroditas protándricos. En espáridos se ha observado que los 2 primeros años de sus vidas maduran y funcionan como machos y al tercer año un porcentaje significativo de los machos (20 cm longitud total) inicia su cambio de sexo a hembra, descompensando de esta forma los lotes establecidos de reproductores (Pavlidis \& Mylonas 2011). En cambio, con el róbalo blanco C. viridis los primeros 3 años funciona como macho y posteriormente inicia su transición de cambio de sexo, pero este cambio de sexo puede durar hasta 3 años más, hasta que alcanzan la talla adecuada (alrededor de $6 \mathrm{~kg}$ ) y se definen como hembras (Datos no publicados). En contraste, con los ejemplares que son hermafroditas protóginos como Pagrus pagrus se ha reportado que pasan a ser machos funcionales a los 3 años. En ese sentido, es de vital importancia conocer el tiempo y/o edad que inician la transición del sexo, conocer los factores que detonan o inhiben su cambio de sexo de manera natural, para poder establecer los protocolos de mantenimiento o renovación de los lotes de reproductores. Para mantener el mismo lote de reproductores, se puede hacer uso de las terapias hormonales; para inhibir o propiciar el cambio de sexo, según lo que se requiera (Piferrer 2001).

Por su aplicabilidad a escala comercial, una de las técnicas más comunes para inducir la reversión sexual en los peces de cultivo y que se utilizan para formar o mantener el lote de reproductores, es la administración de hormonas exógenas a través del alimento (Guiguen et al. 2010, Budd et al. 2015). Sin embargo, en algunos otros peces hermafroditas, se utiliza el factor social como influencia para generar el cambio de sexo o mantener el sexo deseado en los reproductores (Sadovy de Mitchenson \& Liu 2008). Esta estrategia se aplica a diversas especies de meros, como Epinephelus coioides, para poder obtener machos jóvenes con anticipación a su cambio natural (Liu \& Sadovy de Mitchenson 2011, Budd et al. 2015). Otras especies donde influye el factor social por jerarquía es en los peces anemona (payasos), de la familia Amphiprionidae, los cuales son hermafroditas protándricos. Estos peces viven en pequeños grupos sociales el cual consiste en una hembra dominante, generalmente la de mayor tamaño, esta es rodeada por un solo macho y un número variable de juveniles inmaduros de talla pequeña que no se reproducen. Estas especies presentan una fuerte jerarquía social basada en el tamaño, y cuando la hembra dominante del grupo muere o es retirada el macho con mayor jerarquía toma el lugar de la hembra y uno de los juveniles inmaduros toma el lugar del macho, permaneciendo los demás en el mismo estatus (Casas et al. 2016).

\section{MODIFICACIONES DEL PROCESO REPRODUCTIVO DE ESPECIES HERMAFRODITAS EN CAUTIVERIO}

En el desarrollo de la acuicultura marina comercial se requiere tener un completo control de la función reproductiva, para mantener la producción constante de juveniles. Idealmente se desea que los peces se reproduzcan espontáneamente en cautividad, que presenten una alta fecundidad, con una calidad de huevos que permita la producción masiva de juveniles y que el proceso finalice en cultivos de engorda exitosos. Sin embargo, las diferentes especies de peces hermafroditas que se han sometido a condiciones de cautiverio con fines de reproducción controlada, han presentado disfunciones reproductivas, las cuáles se asemejan a las disfunciones reproductivas que presentan varias de las especies gonocóricas que han sido cultivadas a escala comercial (Mylonas \& Zohar 2001). En ambos casos se cree que los problemas son debido a que los peces no experimentan la variedad de influencias externas como en su hábitat natural (Mañanós et al. 2009). Generalmente, en condiciones de cautiverio, las hembras son las que presentan problemas reproductivos más graves. Sin embargo, la disfunción reproductiva dependerá de la especie de cultivo y puede variar desde la ausencia total del desove a reducciones significativas en la cantidad y calidad de huevos y producción de esperma, en el caso de los machos. Lo anterior puede ser debido al estrés causado por el cautiverio, las condiciones de zootecnia y por la ausencia de las señales ambientales apropiadas necesarias para la reproducción (fotoperiodo, temperatura, corrientes, mareas, migraciones, etc.) (Mañanós et al. 2009). Para reducir los problemas reproductivos en las granjas, se debe de proporcionar las mejores condiciones ambientales, nutrición adecuada y técnicas de zootecnia acorde a cada especie. Sin embargo, si los trastornos reproductivos continúan con efectos negativos en el proceso de maduración, entonces el uso de tratamientos hormonales adecuados puede ser la opción para superar los problemas reproductivos, como se ha demostrado en diversos peces cultivados (Mylonas \& Zohar 2001, Zohar \& Mylonas 2001, Mylonas et al. 2011).

Las disfunciones reproductivas que presentan las hembras en condiciones de cautiverio se clasifican en tres tipos. El primer tipo implica la inhibición del proceso de vitelogénesis; en estas especies la reproducción es bloqueada en etapas muy tempranas del desarrollo gonadal. El segundo tipo de disfunción reproductiva es la inhibición 
del proceso de la maduración final del ovocito (FOM). Las especies que presentan este problema se caracterizan porque no completan el proceso de vitelogénesis correctamente, los ovocitos post-vitelogénicos son incapaces de iniciar la FOM y entran en el proceso de atresia, bloqueando cualquier posibilidad de que se obtenga un desove. Este tipo de disfunción reproductiva es la más común detectada en las especies mantenidas en cautiverio, e.g., el róbalo blanco del Pacífico C. viridis, presenta este tipo de disfunción reproductiva (datos no publicados). El tercer tipo de disfunción reproductiva es la inhibición del desove, es decir, las hembras presentan el desarrollo gonadal completo (vitelogénesis, FOM y ovulación), pero el desove es bloqueado y los ovocitos ovulados permanecen en la cavidad abdominal u ovárica y no se liberan al medio (Zohar \& Mylonas 2001). Lo anterior se ha observado en los salmónidos y lenguados, por lo que en estas especies es necesario retirar los ovocitos manualmente (stripping) (Mañanós et al. 2009, Mylonas et al. 2010).

Las disfunciones reproductivas en los machos se consideran generalmente menos importantes que en las hembras y excepto en casos raros, los machos de todas las especies generalmente presentan espermiación en cautiverio. Sin embargo, hay disfunciones reproductivas detectadas, como la disminución del volumen de esperma y de la fluidez del semen, que puede afectar negativamente el proceso de fertilización de los huevos. En los machos de C. viridis y E. labriformis, presentan este tipo de disfunción reproductiva en condiciones de cautiverio (datos no publicados). Finalmente, para contrarrestar las disfunciones reproductivas mencionadas anteriormente, se han utilizado las terapias hormonales. Las terapias hormonales que actualmente se utilizan para la inducción a la maduración final, ovulación, desove y espermiación, han evolucionado a partir de las técnicas denominadas de primera generación desarrolladas en los años 1930s, que involucra las preparaciones a base hormona de la pituitaria de donadores maduros y se homogenizaba en suero fisiológico (Donaldson \& Hunter 1983, Donaldson 1996, Mañanós et al. 2009, Mylonas et al. 2010, Mehdi \& Ehsan 2011). La tecnología de segunda generación involucra el tratamiento hormonal que actúan en el nivel más alto del eje reproductivo, que incluye la hormona liberadora de gonadotropinas del tipo agonista (GnRHa) o agonista de la hormona liberadora de la hormona luteinizante (LHRHa), y en los peces de agua dulce la terapia hormonal se complementa con dopamina antagonista (DA) (Mylonas \& Zohar 2001, Mylonas et al. 2010). Inicialmente, la administración de estas sustancias se realizó mediante inyecciones, logrando la secreción de gonadotropinas hasta por $48 \mathrm{~h}$, pero en algunos organismos no fue tiempo suficiente para inducir la ovulación. La solución para este inconveniente fue aplicar dos o más inyecciones a los organismos después de $24 \mathrm{~h}$ de la primera aplicación, lo que significó un aumento de estrés. Buscando soluciones para evitar la manipulación excesiva de los reproductores, se buscaron nuevos métodos alternativos, que tienen una liberación controlada y continua de la hormona en el sistema circulatorio. Dichos métodos se basan en la utilización de pellets de colesterol o colesterol-celulosa, pellets de copolímeros biodegradables de ácido poliláctico (PLA) y ácido poliglicólico (PGA) o acetato de etileno-vinil (EVAc) (Zohar et al. 1990).

\section{CONCLUSIONES Y PERSPECTIVAS}

La presente revisión señala el marco general del proceso reproductivo de peces hermafroditas y las implicaciones que puede tener el control de su reproducción en cautiverio.

A través de este trabajo se puede inferir que el tratar con especies de peces hermafroditas es más complicado que con especies gonocóricas dado los procesos fisiológicos y morfológicos que deben llevarse a cabo para el cambio de sexo. Sin embargo, también se destaca que puede lograrse la reproducción controlada de este tipo de especies.

Dentro de las repercusiones más fuertes que se pueden presentar en la acuacultura comercial con especies hermafroditas; es la formación de lotes de reproductores (machos y hembras) de la misma edad/talla debido al cambio de sexo, además, esto trae consigo que dicho lote de reproductores cambie su proporción sexual y pueda llegar afectar la fecundidad y por ende la producción controlada de juveniles de un ciclo a otro.

Si el cambio de sexo en especies hermafroditas está influenciado por los esteroides gonadales (e.g., $\mathrm{E}_{2}, 11-\mathrm{KT}$ y T) y existen diversos factores ambientales, sociales o conductuales que desencadenan la producción de estos esteroides; entonces, es importante conocer la fisiología reproductiva del cambio de sexo de cada especie, para controlar su reproducción en cautiverio mediante la estrategia de manejo más adecuada.

La estrategia de manejo más empleada para el cambio de sexo en peces es el uso de terapias hormonales. Esta estrategia se puede emplear para lograr la formación de lotes de reproductores de la misma edad/talla, de esta forma se acelera, se mantiene o induce el cambio de sexo de los organismos deseados. La administración de esteroides sexuales, como $\mathrm{E}_{2}$ y $17 \alpha$-MT son ampliamente utilizados para la feminización y masculinización a nivel comercial.

Este tipo de estrategias puede ser utilizada bajo diferentes métodos de administración de la hormona, los más comunes son por inmersión o impregnado en el alimento. Sin embargo, estos tratamientos son eficientes durante el periodo lábil (final del periodo larvario o inicio del periodo juvenil), por lo que se utilizan normalmente en especies en las que el proceso de diferenciación sexual se produce poco después de la eclosión. Por lo tanto, el cambio de sexo se busca con la finalidad de realizar la etapa de engorda con el sexo que presenta mejores características de crecimiento durante el cultivo. Por otro lado, la aplicación de la hormona en adultos 
también puede ser eficiente, por ejemplo, en el róbalo blanco del Pacífico se logró la obtención de hembras derivados de machos adultos menores de $4 \mathrm{~kg}$ (datos no publicados), usando implantes de $\mathrm{E}_{2}$. Este tipo de método podría ser muy útil para formar o renovar lotes de reproductores con las características deseadas debido a que podría ser posible cambiar de sexo prácticamente al número de peces adultos que se desee, lo cual permite un mayor control de la proporción de sexos del lote de reproductores.

En la actualidad, existe el interés de cultivar diversas especies tropicales y subtropicales como meros, cabrillas, róbalos y algunos espáridos, las cuales son hermafroditas. Sin embargo, aún no existe información suficiente en aspectos biológicos y fisiológicos sobre su proceso de reproducción, que permita desarrollar su cultivo a un nivel comercial. Por ende, es necesario que se desarrolle el conocimiento para cada especie sobre los factores que desencadenan el cambio de sexo y la forma de controlar dicho cambio (ambiental, social o inducción hormonal). Lo anterior permitirá diseñar planes de manejo a corto, mediano y largo plazo, para la formación de lotes de reproductores que permita la obtención de huevos, larvas y juveniles de buena calidad y de manera controlada, como ha sucedido con algunos espáridos que se cultivan actualmente a nivel comercial.

\section{Agradecimientos}

Al Consejo Nacional de Ciencia y Tecnología por el soporte al programa de Posgrado de Ciencias Biologicas de la Universidad Autonoma de Nayarit y al Programa Cátedras CONACYT, con el Proyecto de Cátedras 3285, que otorgó soporte al tercer autor.

\section{LiTERATURA CITADA}

Abozaid H, S Wessels \& G Horstgen-Schwark. 2012. Elevated temperature applied during gonadal transformation leads to male bias in zebrafish (Danio rerio). Sexual Development 6: 201-209.

Afonso L, G Wassermann \& R Oliveira. 2001. Sex reversal in nile tilapia (Oreochromis niloticus) using a nonsteroidal aromatase inhibitor. Journal of Experimental Zoology 290: 177-181.

Allen Y, A Scott, P Matthiessen, S Haworth, J Thain \& S Feist. 1999. Survey of estrogenic activity in United Kingdom estuarine and coastal Waters and its effects on gonadal development of the flounder Platichthys flesus. Environmental Toxicology and Chemistry 18: 1791-1800.

Alvarez-Lajonchére L \& M Tsuzuki. 2008. A review of methods for Centropomus spp. (snooks) aquaculture and recommendations for the establishment of their culture in Latin America. Aquaculture Research 39: 684-700.

Avise J. 2011. Hermaphroditism: A primer on the biology, ecology, and evolution of dual sexuality, $251 \mathrm{pp}$. Columbia University Press, New York.
Avise J \& J Mank. 2009. Evolutionary perspectives on hermaphroditism in fishes. Sexual Development 3: 152-163.

Babiak J, I Babiak, S Nes, T Harboe, T Haugen \& B Norberg. 2012. Induced sex reversal using an aromatase inhibitor, Fadrozole, in Atlantic halibut (Hippoglossus hippoglossus L.). Aquaculture 324/325: 276-280.

Baroiller J, Y Guiguen \& A Fostier. 1999. Endocrine and environmental aspects of sex differentiation in fish. Cellular and Molecular Life Sciences 55: 910-931.

Basurco B, A Lovatelli \& B García. 2011. Current status of sparidae aquaculture, Chapter 1. In: Pavlidis M \& C Mylonas (eds). Sparidae: biology and aquaculture of gilthead sea bream and other species, pp. 1-50. Wiley-Blackwell, Oxford.

Bell J \& M Gervis. 1999. New species for coastal aquaculture in the tropical Pacific- constraints, prospects and considerations. Aquaculture International 7: 207-223.

Blázquez M, A González, M Papadaki, C Mylonas \& F Piferrer. 2008. Sex-related changes in estrogen receptors and aromatase gene expression and enzymatic activity during early development and sex differentiation in the European sea bass (Dicentrarchus labrax). General and Comparative Endocrinology 158: 95-101.

Bromage N \& R Cumaranatunga. 1988. Egg production in the rainbow trout. In: Muir J \& R Roberts (eds). Recent advances in aquaculture, pp. 63-138. Springer, Dordrecht.

Brulé T, C Déniel, T Colás-Marrufo \& M Sánchez-Crespo. 1999. Red grouper reproduction in the southern Gulf of Mexico. Transaction of the American Fishes Society 128: 385-402.

Budd A, Q Banh, J Domingos \& D Jerry. 2015. Sex control in fish: Approaches, challenges and opportunities for aquaculture. Journal of Marine Science and Engineering 3: 329-355.

Casas L, F Saborido-Rey, T Ryu, C Michell, T Ravasi \& X Irigoien. 2016. Sex change in clowfish: Molecular insights from transcriptome analysis. Scientific Reports 6: 35461.

Chang C, M Lee \& G Chen. 1994. Estradiol-17 $\beta$ associated with the sex reversal in protandrous black porgy, Acanthopagrus schlegeli. The Journal of Experimental Zoology 268: 53-58.

Cheshenko K, F Pakdel, H Segner, O Kah \& R Eggen. 2008. Interference of endocrine disrupting chemicals with aromatase CYP19 expression or activity, and consequences for reproduction of teleost fish. General and Comparative Endocrinology 155: 31-62.

Chichizola C. 2004. Disruptores endocrinos. Efectos en la reproducción. Revista Argentina de Endocrinología y Metabolismo 41: 79-105.

Colborn T, F Saal \& A Soto. 1993. Developmental effects of Endocrine-Disrupting Chemicals in wildlife and humans. Environmental Health Perspectives 101(5): 378-384.

DeRosa C, P Richter, H Pohl \& D Jones. 1998. Environmental exposures that affects the endocrine system: Public health implications. Journal of Toxicology and Environmental Health, Part B 1: 3-26.

Devlin R \& Y Nagahama. 2002. Sex determination and sex differentiation in fish: an overview of genetic, physiological, and environmental influences. Aquaculture 208: 191-364.

Donaldson E. 1996. Manipulation of reproduction in farmed fish. Animal Reproduction Science 42: 381-392. 
Donaldson E \& G Hunter. 1983. Induced final maturation, ovulation and spermiation in cultured fish. In: Hoar W, D Randall \& E Donaldson (eds). Fish physiology. Volumen IX Reproduction, Part B, Behavior and fertility control, pp. 351-403. Academic Press, New York.

Folmar L, N Denslow, V Rao, M Chow, D Crain, J Enblom, J Marcino \& L Guillette Jr. 1996. Vitellogenin induction and reduced testosterone concentrations in feral male carp (Cyprinus carpio) capture near a major metropolitan sewage treatment plant. Environmental Health Perspectives 104: 1096-1101.

Folmar L, N Denslow, K Kroll, E Orlando, J Emblom, J Marcino, C Metcalfe \& L Guillette Jr. 2001. Altered serum sex steroids and vitellogenin induction in walleye (Stizostedion vitreum) collected near a metropolitan sewage treatment plant. Archives of Environmental Contamination and Toxicology 40: 392-398.

Frisch A. 2004. Sex-change and gonadal steroids in sequentiallyhermaphroditic teleost fish. Reviews in Fish Biology and Fisheries 14: 481-499.

Godwin J \& P Thomas. 1993. Sex change and steroid profiles in the protandrous anemonefish Amphiprion melanopus (Pomacentridae, Teleostei). General and Comparative Endocrinology 91: 144-157.

Guiguen Y, B Jalabert, E Thouard \& A Fostier. 1993. Changes in plasma and gonadal steroid hormones in relation to the reproductive cycle and the sex inversion process in the protandrous sea bass, Lates calcarifer. General and Comparative Endocrinology 92: 327-338.

Guiguen Y, C Cauty, A Fostier, J Fuchs \& B Jalabert. 1994. Reproductive cycle and sex inversion of seabass, Lates calcarifer, reared in sea cages in French Polynesia. Histological and morphometric description. Environmental Biology of Fishes 39: 231-247.

Guiguen Y, J Baroiller, M Ricordel, K Iseki, O McMeel, S Martin \& A Fostier. 1999. Involvement of estrogens in the process of sex differentiation in two fish species: The rainbow trout (Oncorhynchus mykiss) and a tilapia (Oreochromis niloticus). Molecular Reproduction and Development 54: 154-162.

Guiguen Y, A Fostier, F Piferrer \& C Chang. 2010. Ovarian aromatase and estrogens: A pivotal role for gonadal sex differentiation and sex change in fish. General and Comparative Endocrinology 165: 352-366.

Hamlin H. 2014. Endocrine disruption. In: Evans D, J Claiborne \& S Currie (eds). The physiology of fishes, pp. 235-255. Taylor and Francis, Boca Raton.

Hayashi Y, H Kobira, T Yamaguchi, E Shiraishi, T Yazawa, T Hirai, Y Kamei \& T Kitano. 2010. High temperature causes masculinization of genetically female medaka by elevation of cortisol. Molecular Reproduction and Development 77: 679-686.

Hinfray N, J Porcher \& F Brion. 2006. Inhibition of rainbow trout (Oncorhynchus mykiss) P450 aromatase activities in brain and ovarian microsomes by various environmental substances. Comparative Biochemistry and Physiology Part C: Toxicology and Pharmacology 144: 252-262.

Holland B, G Monteverdi \& R Di Giulio. 1999. Octyphenol induces vitellogenin production and cell death in hepatocytes. Environmental Toxicology and Chemistry 18(4): 734-739.
Jones Y, F Lee, W Yueh, P Tacon, J Du, C Chang, S Jeng, H Tanaka \& C Chang. 2000. Vitellogenin as a biomarker for environmental estrogens. Water Science and Technology 42: 1-14.

Kavlock R, G Daston, C DeRosa, P Fenner-Crisp, L Gray, S Kaattari, G Lucier, M Luster, M Mac, C Maczka, R Miller, J Moore, R Rolland, G Scott, D Sheehan, T Sinks \& H Tilson. 1996. Research needs for the risk assessment of health and environmental effects of endocrine disruptors: A report of the U.S. EPA-sponsored workshop. Environmental Health Perspectives 4: 715-740.

Lance V. 2009. Is regulation of aromatase expression in reptiles the key to understanding temperature-dependent sex determination? Journal of Experimental Zoology 311A: 314-322.

Lee Y, J Du, W Yueh, B Lin, J Huang, C Lee \& M Lee. 2001. Sex change in the protandrous black porgy Acanthopagrus schlegeli: A review in gonadal development, estradiol, estrogen receptor, aromatase activity and gonadotropin. Journal of Experimental Zoology 290: 715-726.

Li G, X Liu \& H Lin. 2007. Seasonal changes of serum sex steroids concentration and aromatase activity of gonad and brain in red-spotted grouper (Epinephelus akaara). Animal Reproduction Science 99: 156-166.

Liu M \& Y Sadovi de Mitcheson. 2011. The influence of social factor son juvenile sexual differentiation in a diandric, protogynous grouper Ephinephelus coioides. Ichthyological Research 58: 84-89.

Mañanós E, N Duncan \& C Mylonas. 2009. Reproduction and control of ovulation, spermiation and spawning in cultured fish. In: Cabrita E, V Robles \& M Herráez (eds). Methods in reproductive aquaculture: Marine and freshwater species, pp. 3-80. CRC Press, Boca Raton.

Mehdi Y \& M Ehsan. 2011. A review of the control of reproduction and hormonal manipulations in finfish species. African Journal of Agricultural Research 6(7): 1643-1650.

Mills L \& C Chichester. 2005. Review of evidence: Are endocrine-disrupting chemicals in the aquatic environment impacting fish populations? Science of the Total Environment 343: 1-34.

Munday P, M Caley \& G Jones. 1998. Bi-directional sex change in a coral-dwelling goby. Behavioral Ecology and Sociobiology 43(6): 371-377.

Mylonas C \& Y Zohar. 2001. Use of GnRHa-delivery systems for the control of reproduction in fish. Reviews in Fish Biology and Fisheries 10: 463-491.

Mylonas C,A Fostier \& S Zanuy. 2010. Broodstock management and hormonal manipulations of fish reproduction. General and Comparative Endocrinology 165: 516-534.

Mylonas C, Y Zohar, N Pankhurst \& H Kagawa. 2011. Reproduction and broodstock management. In: Pavlidis M \& C Mylonas (eds). Sparidae: biology and aquaculture of gilthead seabream and related species, pp. 95-131. Blackwell Publishing, London.

Nakamura M, T Kobayashi, X Chang \& Y Nagahama. 1998. Gonadal sex differentiation in teleost fish. Journal of Experimental Zoology 281: 362-372.

Pandian T. 2010. Sexuality in fishes, 218 pp. Science Publishers/ CRC Press, Enfield/Boca Raton. 
Pandian T. 2011. Sex determination in fish, 294 pp. Science Publishers/CRC Press, Enfield /Boca Raton.

Pandian T. 2013. Endocrine sex differentiation in fish, 318 pp. CRC Press, Boca Raton.

Pandian T \& S Sheela. 1995. Hormonal induction of sex reversal in fish. Aquaculture 138: 1-22.

Patiño R, G Yoshizaki, T Peter \& H Kagawa. 2001. Gonadotropic control of ovarian follicle maturation: the two-stage concept and its mechanisms. Comparative Biochemistry and Physiology Part B 129: 427-439.

Pavlidis M \& C Mylonas. 2011. Sparidae: Biology and aquaculture of Gilthead Sea Bream and other species, 390 pp. Wiley-Blackwell, Oxford.

Piferrer F. 2001. Endocrine sex control strategies for the feminization of teleost fish. Aquaculture 197: 229-281.

Piferrer F. 2009. Determinación y diferenciación sexual en los peces. En: Carrillo M (ed). La reproducción de los peces: aspectos básicos y sus aplicaciones en acuicultura, pp. 249-278. Fundación Observatorio Español de Acuicultura, Madrid.

Piferrer F \& M Blázquez. 2005. Aromatase distribution and regulation in fish. Fish Physiology and Biochemistry 31: 215-226.

Piferrer F \& Y Guiguen. 2008. Fish gonadogenesis. Part II: Molecular biology and genomics of sex differentiation. Review in Fisheries Science 16S1: 35-55.

Piferrer F, S Zanuy, M Carrillo, I Solar \& R Devlin. 1994. Brief treatment with aromatase inhibitor during sex differentiation causes chromosomally female salmon to develop as normal functional males. The Journal of Experimental Zoology 270: 255-262.

Sadovy de Mitchenson Y \& M Liu. 2008. Functional hermaphroditism in teleosts. Fish and Fisheries 9: 1-43.

Schulz RW, L Renato de Franca, J-J Lareyre, F LeGac, H Chiarini-Garcia, RH Nobrega \& T Miura. 2010. Spermatogenesis in fish. General and Comparative Endocrinology 165: 390-411.

Segner H, K Caroll, M Fenske, C Janssen, G Maack, D Pascoe, C Schäfers, G Vandernbergh, M Watts \& A Wenzel. 2003. Identification of endocrine-disrupting effects in aquatic vertebrates and invertebrates: Report form the European IDEA Project. Ecotoxicology and Environmental Safety 54: 302-314.

Shen Z-G, Q-X Fan, W Yang, Y-L Zhang, P-P Hu \& C-X Xie. 2013. Effects of non-steroidal aromatase inhibitor letrozole on sex inversion and spermatogenesis in yellow catfish Pelteobagrus fulvidraco. The Biological Bulletin 225: 18-23.

Struussmann C, S Moriyama, E Hanke, J Calsina \& F Takashima. 1996. Evidence of thermolabile sex determination in pejerrey. Journal of Fish Biology 48: 643-651.
Sun L, R Jin, Z Peng, Q Zhou, H Qian \& Z Fu. 2014. Effects of trilostane and fipronil on the reproductive axis in an early life stage of the Japanese medaka (Oryzias latipes). Ecotoxicology 23(6): 1044-1054.

Tan S \& K Tan. 1974. Biology of tropical grouper Epinephelus tauvina (Forskal). A preliminary study on hermaphroditism Epinephelus tauvina. Singapore Journal of Primary Industries 2: 123-133.

Taylor R, H Grier \& J Whittington. 1998. Spawning rhythms of common snook in Florida. Journal of Fish Biology 53: 502-520.

Taylor R, J Whittington, H Grier \& R Crabtree. 2000. Age, growth, maturation and protandric sex reversal in common snook, Centropomus undecimalis, from the east and west coasts of Florida. Fishery Bulletin 98: 612-624.

Tzchori I, T Zak \& O Sachs. 2004. Masculinization of genetic females of the common carp (Cyprinus carpio L.) by dietary administration o fan aromatase inhibitor. The Israeli Journal of Aquaculture-Bamidgeh 56(4): 239-246.

Valdivia K, E Jouanno, J Volff, D Galiana-Arnoux, R Guyomard, L Helary, B Mourot, A Fostier, E Quillet \& Y Guiguen. 2014. High temperature increases the masculinization rate of the all-female (XX) rainbow trout "Mal" population. PloS ONE 9(12): e113355. < https://doi. org/10.1371/journal.pone.0113355>

Wallace R \& K Selman. 1990. Ultrastructural aspects of oogenesis and oocyte growth in fish and amphibians. Journal of Electron Microscopy Technique 16: 175-201.

Wong T, S Ijiri \& Y Zohar. 2006. Molecular biology of ovarian aromatase in sex reversal: Complementary DNA and 5 '-Flanking region isolation and differential expression of ovarian aromatase in the gilthead seabream (Sparus aurata). Biology of Reproduction 74: 857-864.

Wootton J \& C Smith. 2015. Reproductive biology of teleost fishes, 499 pp. John Wiley \& Sons, Hoboken.

Yamamoto T. 1969. Sex differentiation. In: Hoar W \& D Randall (eds). Fish Physiology 3: 117-175. Academic Press, New York.

Yaron Z \& B Sivan. 2005. Reproduction. In: Evans D \& J Clairbone (eds). The physiology of fishes, pp. 343-386. Science Publishers/CRC Press, Enfield /Boca Raton.

Zohar Y \& C Mylonas. 2001. Endocrine manipulations of spawning in cultured fish: from hormones to genes. Aquaculture 197: 99-136.

Zohar Y, M Abraham \& H Gordin. 1978. The gonadal cycle of the captivity reared hermaphroditic teleost Sparus aurata (L.) during the first two years of life. Annales de Biologie Animale, Biochimie Biophysique 18(4): 877-882.

Zohar Y, G Pagelson, Y Gothilf, W Dickhoff, P Swanson, S Duguay, W Gombotz, J Kost \& R Langer. 1990. Controlled release of gonadotropin releasing hormones for the manipulation of spawning in farmed fish. Controlled Release of Bioactive Materials 17: 51-52. 